\title{
Comparison between Adventitial and Intimal Inflammation of Ruptured and Nonruptured Atherosclerotic Plaques in Human Coronary Arteries
}

\author{
Maria L. Higuchi, Paulo S. Gutierrez, Hiram G. Bezerra, Suely A. Palomino, Vera D. Aiello, \\ Júlia M. L. Silvestre, Peter Libby, José A. F. Ramires
}

São Paulo, SP - Brazil

\begin{abstract}
Objective - To verify the possible role of adventitial inflammation in atherosclerotic plaque vulnerability and coronary artery remodelling.
\end{abstract}

Methods - We compared the mean numbers of lymphocytes in the adventitia and in the plaque of ruptured thrombosed and stable equi-stenotic coronary segments of 34 patients who died due to acute myocardial infarction. We also analysed adventitial microvessels, adventitial fibrosis and the external elastic membrane.

Results - In the adventitia, the numbers of lymphocytes and microvessels $/ \mathrm{mm}^{2}$ were $69.5 \pm 88.3$ and $60.9 \pm 32.1$ in culprit lesions and $16.4 \pm 21.1$ and $44.3 \pm 16.1$ in stable lesions ( $p<0.05$ ); within the plaques, the mean number of lymphocytes was $24 \pm 40.8$ in culprit lesions and $10.9 \pm 13.2$ in stable ones $(p=0.17)$. The mean percent area of adventitial fibrosis/ cross-sectional area of the vessel was significantly lower in unstable plaques $(p<0.001)$. The confocal images showed holes in the external elastic membrane.

Conclusion - Unstable plaques exhibit chronic panarteritis, accompanied by enlargement, medial thinning, and less fibrosis than in stable lesions, which is compatible with vessel aneurysm. Adventitial inflammation may contribute significantly to atheroma instability.

Key words: coronary atherosclerosis, unstable plaques, arteritis, aneurysm, adventitial inflammation

Heart Institute (InCor), University of São Paulo Medical School, São Paulo, Brazil and Cardiovascular Division, Brigham and Women's Hospital, Boston, MA, USA Mailing address: Maria de Lourdes Higuchi - InCor - Av. Dr. Enéas C. Aguiar, 44 05403-000 - São Paulo, SP - Brasil - E-mail: anplourdes@incor.usp.br Received for publication on $6 / 28 / 01$

Accepted on $9 / 12 / 01$
Much experimental and clinical evidence links inflammation and atherosclerosis; for example, systemic indices of inflammation may play a role in predicting risk of coronary events $^{1,2}$. Many reports ${ }^{3-8}$ have attributed to inflammation in the cap and shoulder of the atherosclerotic lesion a fundamental pathogenetic role in plaque disruption and consequent acute thrombotic complications; otherwise, microvessels in atherosclerotic plaques have been considered to favor the influx of inflammatory cells into them ${ }^{9,10}$. Few pathological studies have addressed the involvement of inflammation in the media or adventitia ${ }^{11,12}$ in the development of atherosclerosis and arterial remodelling. We have recently demonstrated that unstable thrombosed plaques responsible for fatal acute myocardial infarction (AMI) are usually larger than equi-stenotic stable plaques in the same patient, having larger lumina due to the compensatory positive remodelling of the vessels ${ }^{13}$. In the present study, we propose that the chronic adventitial inflammation and neovascularization may contribute to the thinning of the medial layer and compensatory enlargement of coronary segments with vulnerable atherosclerotic plaques.

\section{Methods}

The present work was approved by the Scientific and Ethics Committee of the Heart Institute (InCor) of São Paulo University Medical School.

Thirty-four specimens from consecutive necropsies performed at the Heart Institute (InCor) of the University of São Paulo Medical School from patients who died due to AMI from 1985 to 1986 were studied. This period was chosen because at that time procedures like angioplasty or thrombolysis were not a routine in this hospital, thus the coronary arteries were free of conditions that could produce artificial changes in plaque morphology. All but 2 specimens had been used in a previous study from our group ${ }^{13}$.

Cross sections from 2 groups of coronary artery seg- 
ments were analyzed: Group I - 34 thrombosed coronary artery segments responsible for the AMI; and Group II - 34 nonruptured (stable) segments situated in another coronary artery branch of the same heart, with a similar grade of stenosis and matched according to the distance from the coronary ostium.

Tissue blocks containing the culprit lesions were serially sectioned to define the exact site of plaque rupture or erosion. At each interval of $30 \mu \mathrm{m}$, we reserved $5 \mu \mathrm{m}$-thick sections for histological analysis. The section exhibiting the most extensive plaque rupture was used for morphometry of the vessel areas and plaque constitution. Subsequent sections were used to quantify the inflammatory cells, adventitial microvessels, and thicknesses of the medial layer, adventitia, and external elastic membrane (EEM).

Atherosclerotic plaque areas - We used the data obtained in the previous study ${ }^{13}$, presented in table 1: a Leica image analysis Quantimet 500 system was used to measure the plaque area (the region encompassed by the elastic internal membrane) in Movat-stained coronary artery histological cross sections. Group I had larger plaques $\left(9.8 \pm 4.8 \mathrm{~mm}^{2}\right)$ than $\operatorname{didGroup~II}\left(4.8 \pm 2.3 \mathrm{~mm}^{2}\right)$.

Microvessels present in all tangential 400x microscopic fields that surrounded the arterial cross section were counted in Movat-stained slides.

Immunohistochemistry was used for characterization of the CD8-T, CD4-T, and CD20-B cells, with Novocastra, UK (CD8) and Dako-Patts (CA, USA) antibodies. For CD8epitope recovery, the sections were boiled in sodium citrate buffer ( $10 \mathrm{mM}, \mathrm{pH} 6.0)$ for 15 seconds before reactions. Immunohistochemical detection of the epitopes used the indirect horseradish peroxidase technique as previously described ${ }^{14}$.

The lymphocytes present in all 400x microscopic fields of the adventitia were counted in each of the selected segments. The total amounts were divided by the number of fields and divided by $0.36 \mathrm{~mm}^{2}$ (which is the area of each microscopic field) to obtain the mean adventitial number of lymphocytes $/ \mathrm{mm}^{2}$.

Lymphocytes located within the plaques were also

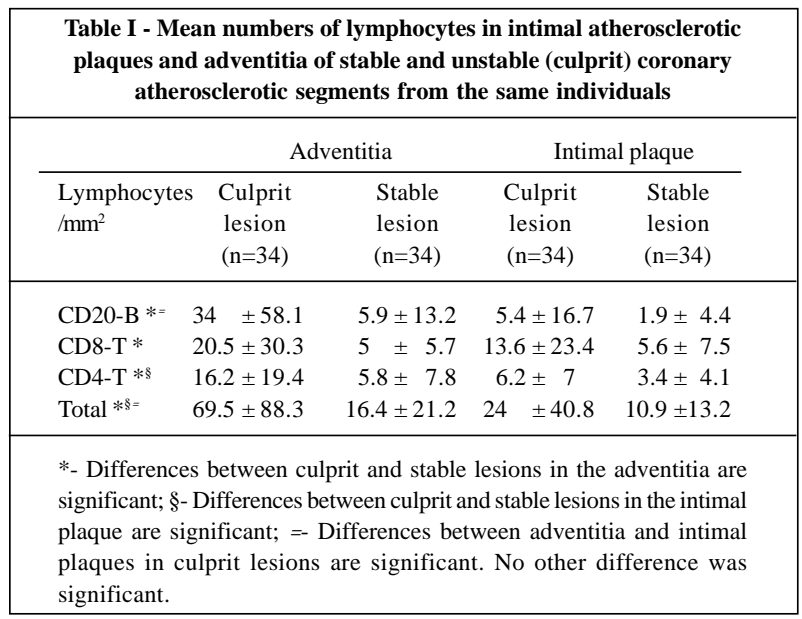

counted; their numbers were divided by the area of the plaque (discounting the fat area, which was devoid of lymphocytes) to obtain the plaque concentration of lymphocytes $/ \mathrm{mm}^{2}$.

The areas of adventitial fibrosis and the medial layer were measured using the Leica Image Analysis System Quantimet 500, in Masson's trichrome-stained sections. These results were divided by the respective cross-sectional area of the vessel from the EEM, thus indicating the relative thickness of these components. Movat's stain was used to identify the elastic membranes. The mean of 3 measures in 400x microscopic magnification of EEM thickness and of adventitial fibrosis juxtaposed to the EEM were obtained, both at the site where the atheroma was more developed and at the opposite side. For measurement of EEM thickness, we considered only the closely disposed elastic fibers. Distant fragmented elastic fibers mixed into the fibrosis were not included in the thickness of the EEM. Qualitative evaluation of EEM was performed in 3-D images with confocal laser microscopy, in $20 \mu \mathrm{m}$-thick sections stained with antinuclei fluorescent reagent (bath with propidium iodide [Sigma, MO, USA] at 1:400 during 3 minutes in a humid chamber at dark). Because the elastic membrane is auto-fluorescent and detected in a fluorescein channel, 2 color images could be acquired.

Statistical analysis - The comparisons concerning quantitative data were performed with Friedman's rank test. Pearson's test was used for the correlation between percentage of adventitial fibrosis and cross-sectional area of the vessel. Tests with $p \leq 0.05$ were considered significant.

\section{Results}

The mean number of adventitial microvessels $/ \mathrm{mm}^{2}$ was $60.9 \pm 32.1$ in group I (coronary artery segments with culprit lesions) and $44.3 \pm 16.1$ in group II (with stable atherosclerotic plaques $)(\mathrm{p}=0.04)$.

The quantitative data concerning lymphocytes are summarized in table I; figure 1 is a graphic representation of the amounts of total lymphocytes.

In the adventitia, the mean numbers of CD20-B, CD8-T, CD4-T, and total lymphocytes $/ \mathrm{mm}^{2}$ were significantly higher in the adventitial layer of Group I than in Group II $(\mathrm{p}<0.001$ for all of them; table I). The CD20-B cell was the main lymphocyte present in the adventitia of Group I; in Group II, a similar proportion existed among the 3 subtypes of lymphocytes.

Within the plaques (excluding the lipidic area), the difference between the 2 groups was not significant with regard to the mean number of total lymphocytes $(24 \pm 40.8$ in Group I vs 10.9 \pm 13.2 in Group II, $\mathrm{p}=0.17$ ), CD8T cell - which was the main lymphocyte present (13.6 \pm 23.4 and 5.6 \pm 7.5 , $\mathrm{p}=0.09)$, and CD20-B (5.4 $\pm 16.7 \mathrm{vs} 1.9 \pm 4.4, \mathrm{p}=0.49)$, but more CD4T cells were present in the culprit (mean 6.2 \pm 7 ) than in the stable lesions $(3.4 \pm 4.1)(\mathrm{p}=0.04)$.

Comparing plaque versus adventitia in the same segments, in Group I more CD20B and total lymphocytes $/ \mathrm{mm}^{2}$ were present in the adventitia than in the plaque $(34 \pm 58.1 \mathrm{vs}$ 


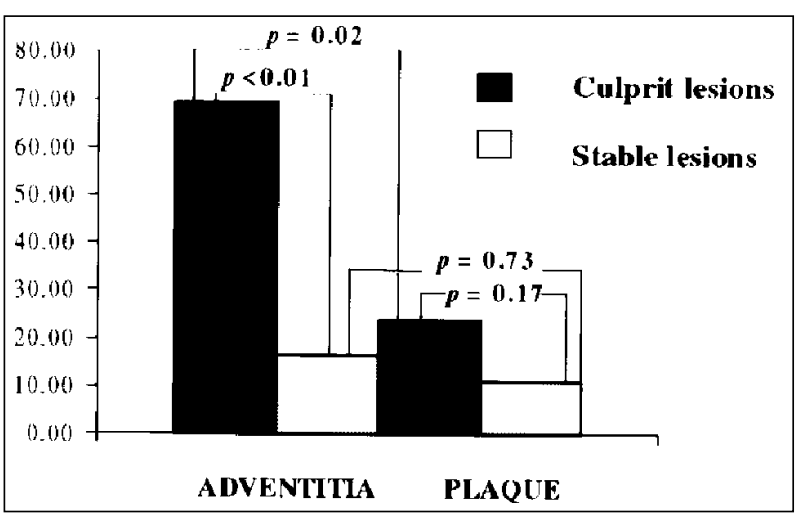

Fig. 1. Number of lymphocytes $/ \mathrm{mm}^{2}$ in the adventitia and atherosclerotic plaques of atherosclerotic plaques and adventitia of stable and unstable (culprit) coronary atherosclerotic segments from the same individuals

$5.4 \pm 16.7, \mathrm{p}<0.01$, and $69.5 \pm 88.3$ vs $24 \pm 40.8, \mathrm{p}=0.02)$; the differences concerning CD8T $(20.5 \pm 30.3$ vs $13.6 \pm 23.4)$ and CD4T (16.2 \pm 19.4 vs 6.2 \pm 7 ) were not significant ( $\mathrm{p}=0.28$ and $\mathrm{p}=0.06$, respectively). In the stable plaques (Group II), none of the differences were significant (CD20B- 5.9 \pm 13.2 vs $1.9 \pm 4.4, \mathrm{p}=0.17$; CD8T- 5.0 \pm 5.7 vs 5.6 $\pm 7.5, \mathrm{p}=0.6$; CD4T$5.8 \pm 7.8$ vs $3.4 \pm 4.1, \mathrm{p}=0.39$; total lymphocytes $-16.4 \pm 21.2 \mathrm{vs}$ $10.9 \pm 13.2, \mathrm{p}=0.73)$.

Neutrophils were almost absent in stable plaques and present in variable amounts in ruptured plaques, mainly adjacent to the thrombi but never as an important component of the inflammation. Macrophages were the most frequent inflammatory cells, in the ruptured plaques, usually full of intracytoplasmic lipids (foam cells). Variable amounts of plasma cells were seen adjacent to the other types of lymphocytes, mainly in the adventitia.

The mean percent area of adventitial fibrosis/crosssectional area of the vessel was significantly lower in Group I than in Group II (16.24 $\pm 5.07 \%$ vs $28.95 \pm 9.76 \%$ respectively; $\mathrm{p}<0.001)$. A weak negative linear correlation existed between percentage of adventitial fibrosis and cross-sectional area of the vessel $(\mathrm{r}=-0.57 ; \mathrm{p}<0.01)$. The mean percent area of medial layer/cross-sectional area of the vessel was significantly lower in Group I than in Group II $(11.79 \pm 4.35 \%$ vs $20.9 \pm 8.45 \%, \mathrm{p}<0.001)$. Figure 2 exemplifies such medial attenuation that occurs in vulnerable plaques; associated with mononuclear inflammation of the wall.

The EEM under the base of the ruptured plaque was thinner than that of the stable plaques $(3.81 \pm 2.59 \mathrm{vs}$ $6.87 \pm 5.49 \mu \mathrm{m}, \mathrm{p}=0.003)$. The EEM on the base of the unstable plaques was more fragmented and thinner (fig. 2B); a significant difference existed between the thickness of the EEM at the side of the major plaque burden (where the EEM was thinner) than at the opposite side. It was more evident in Group I but also occurred in Group II ( $3.81 \pm 2.59$ vs $7.13 \pm 7.32$, $\mathrm{p}=0.005$; and $6.87 \pm 5.49$ vs $8.47 \pm 5.97, \mathrm{p}=0.06)$. However, in Group II, the EEM was less fragmented, and usually thickened (fig. 2C). The 3-D confocal images show holes in the external elastic membranes and inflammatory cells surrounding them (fig. 2D), in a thrombosed ruptured plaque segment.

\section{Discussion}

The role of inflammation in atherosclerosis could involve not only the pathogenesis of atherosclerotic plaques, but also their rupture. Concerning this last point, the intima has received much attention as a site of inflammation ${ }^{1-6}$, while the adventitia has remained relatively unexplored.

In the present study, we compared ruptured plaques with stable ones. We chose lesions from the same patients, at a similar distance from the beginning of the coronary branch, and causing approximately the same degree of stenosis. Thus, the differences that would be found were probably related to the acute complication. We morphologically quantified some features that denote inflammation, namely the number of lymphocytes and microvessels, comparing the variations between ruptured and stable plaques both in the intimal lesions and in the corresponding adventitial zones. Interestingly, the differences were more prominent in the adventitia, where there was a significantly higher number of lymphocytes/mm² (due mostly to CD20-B cells) than in the plaques in the unstable group.

In an ongoing study, we compared another group of obstructive stable atheromatous coronary artery segments. These are from patients submitted to elective myocardial revascularization who died due to causes other than acute myocardial infarction. No significant histopathological differences was found with the present group II of stable plaques $^{27}$.

With ultrasound analysis, Depre et $\mathrm{al}^{15}$ found more neovessels in the plaques of patients with acute syndromes than in patients with stable coronary insufficiency. Kumamoto et al ${ }^{16}$ observed that the density of new intimal vessels correlated with the incidence of lumen stenosis and severity of inflammatory infiltrate. Zhang et al ${ }^{17}$ demonstrated a direct correlation between the increased amount of intimal microvessels and intimal thickness. Our findings agree with these data and may reflect increased angiogenic stimulation from lymphocytes. In this regard, activation can induce $T$ cells to develop angiogenic mediators ${ }^{18}$. In spite of predominating B cells, a great amount of T cells were present in the adventitia. The stimulus for B cells could be the presence of bacteria as we have demonstrated in a recent article ${ }^{3}$. The most numerous inflammatory cell in the plaque is the macrophage, mainly in unstable plaques, as we have already reported ${ }^{13}$. However, apparently, macrophages are performing a phagocytic function because they are usually full of lipids (foam cells). In the adventitia, macrophages were also present but in small quantities.

To better understand the role of adventitial inflammation in atherosclerosis, it is worth calling attention to the fact that, as we showed in another study using the same patient group ${ }^{13}$, as well as by intravascular ultrasound imaging ${ }^{20}$, the ruptured plaques are bigger than stable lesions, and the segments containing them are dilated. Associating the present findings with the previous ones, we propose that rupture of coronary artery plaques usually occur in huge lipid plaques that form in an aneurysmatic dilated segment of the vessel as a consequence of pan-arteritis (fig. 3). 


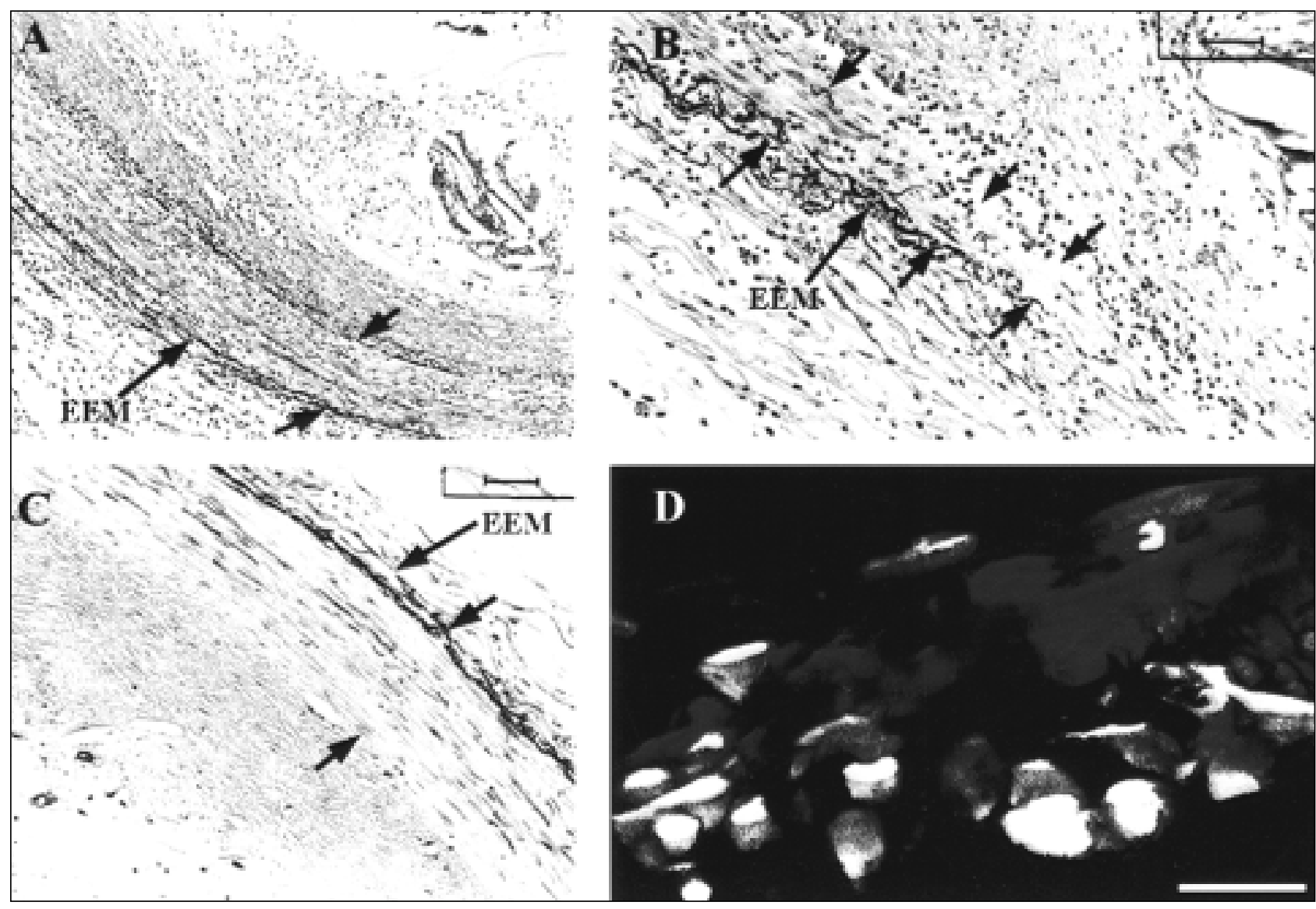

Fig. 2. A) Histological view of a thrombosed ruptured atherosclerotic plaque showing large lipid core, attenuation of the media (between small arrows) and severe inflammation of the adventitia, which extends to the media and to the base of the plaque. The external elastic membrane (EEM - long arrow) is fragmented. (Movat- 100x). B) Detailed histological view of a ruptured plaque vascular segment exhibiting progressive disappearance of the media (between small arrows) and fragmentation of the external elastic membrane (EEM long arrow), associated with inflammatory cells and neovascularization in the adventitia. The inflammatory infiltrate reaches the base of the plaque (Movat - 200x). C) Comparative detailed histological picture of a stable plaque vessel segment exhibiting well preserved media (between small arrows), thickened external elastic membrane (EEM - long arrow), and lack of adventitial inflammation (Movat 200x). D) 3D microscopic view at the confocal laser microscopy showing lymphocytes in yellow in close contact with a external elastic membrane (red) presenting many holes (white arrows) in a vulnerable segment.

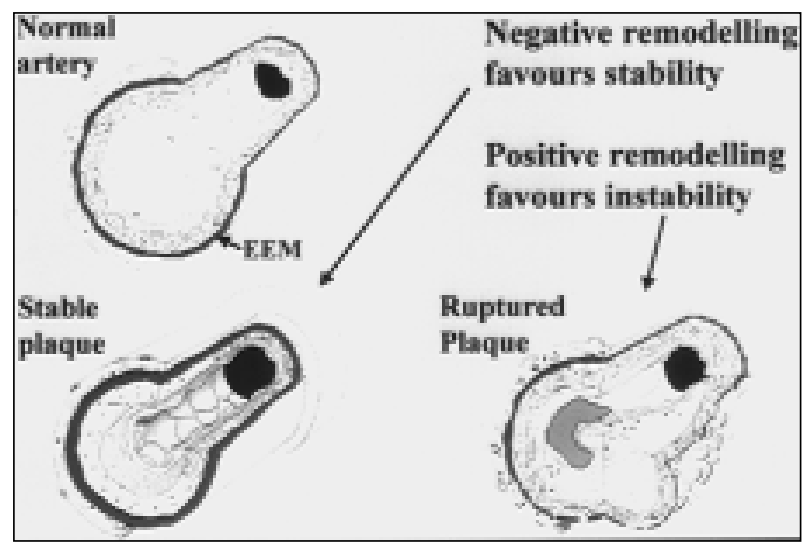

Fig. 3 - Sketch representing different scenarios for lumen atherosclerotic obstruction determined by different forms of vessel remodelling. Negative remodelling shows adventitial fibrosis, thickenning of external elastic membrane (EEM - in red), associated with medial layer hypertrophy. The plaque is fibrocellular, containing many smooth muscle cells; even if its area is small, it may cause severe obstruction of the lumen. Positive remodelling is associated to adventitial inflammation with less fibrosis, fragmentation of EEM, medial thinning and vessel dilatation; the plaque is large, contains more lipid, is more vulnerable and the lumen may be preserved.

Although atherosclerosis is common in the abdominal aorta, aneurysms are quite infrequent. Many articles sug- gest that a great number of the elastic fibers must be destroyed for this type of lesion to occur ${ }^{21,22}$. On the other hand, the main source of elastase, as well as of the other metalloproteinases that degrade extracellular matrix proteins, are the inflammatory cells ${ }^{23}$. In accordance with our hypothesis that unstable lesions could be arteritis with aneurysmatic dilatation, aortic atherosclerotic aneurysms are also associated with a pronounced inflammation, composed mostly of plasma cells ${ }^{24}$, and infectious agents such as Chlamydia pneumoniae have also been found ${ }^{25}$.

Our hypothesis is consistent with data in the literature indicating that B cells are important for stimulating CD40L (CD154) in atherogenesis ${ }^{26}$. In the present study, we observed a prominent B cell (CD20+lymphocyte) infiltrate in the adventitia. Thus, we measured the external elastic membrane in silver-stained sections with common microscopy to check whether areas with severe inflammation have elastolysis and dilate. This membrane was thinner in the regions of the artery containing the greatest atherosclerotic plaques than in the remaining portions of the vessel; importantly, this difference was more prominent in the unstable segments (mean of $3.8 \mu \mathrm{m}$ in the region of the ruptured plaque 
and $7.1 \mu \mathrm{m}$ at the opposite side of the same segment; mean of $6.9 \mu \mathrm{m}$ versus $8.5 \mu \mathrm{m}$ in the control segments). The confocal laser microscopical analysis confirmed these findings.

Rupture of the EEM also occurred on the base of stable plaques, but to a lesser degree than on the unstable ones. We speculate that in the beginning of the lipidic deposition process, vessel wall inflammation is already present. The healing of such inflammatory lesions, to circumscribe the lipid accumulation, would lead to development of stable atheromas, characterised not only by a fibrotic cap on the intimal surface, but also on the base and on the adjacent adventitia, explaining also the EEM fragmentation. This fibrotic healing tissue may cause vessel constriction (negative remodelling), also explaining severe obstruction caused by small fibrotic plaques (fig. 3 ).

It is noteworthy and traditionally reported that thrombosis is not frequent in the aorta, even with severe atherosclerosis, but is commonly found in aortic aneurysms. The usual explanation to for this is that in aneurysmatic areas the blood flow is slow. A role for fibrous cap degradation, linked to the inflammatory process, could be taught is these cases as well.

In conclusion, a contrast can be noted between areas containing ruptured and stable atherosclerotic plaques (fig. 3). Segments with stable plaques display either narrowing or absence of remodelling. In these lesions, the vessel shows medial thickening. Due to the whole vessel diameter narrowing, even small coronary artery intimal plaques could substantially obstruct the lumen. Unstable atherosclerosis would usually be related to a greater inflammatory infiltrate and increased number of microvessels at the adventitia. The involvement of this layer contributes to the characterisation of a process of pan-arteritis, which might be linked, by the action of elastase and other matrix-degrading enzymes ${ }^{22}$, to dilation of the arterial segment (aneurysm). Thus, even large lesions could be associated with only moderate percent coronary obstruction, plaque rupture, and thrombosis.

\section{Acknowledgements}

This research was performed with financial support from FAPESP (São Paulo Foundation of Assistance for Researchers), process \# 99/00322-9 and Fundação E. J.Zerbini. We thank to Argemiro F. Júnior for the coronary schematic design.

\section{References}

1. Ridker PM, Kennekens CH, Buring JE, Rifai N. C-reactive protein and other markers of inflammation in the prediction of cardiovascular disease in women. $\mathrm{N}$ Engl J Med 2000; 342: 836-43.

2. Kario K, Sakata T, Miyata T, Kiechl S, Willeit J. Is acquired activated protein C resistance a cardiovascular risk? Circulation 2000; 101: E120.

3. Boyle JJ. Association of coronary plaque rupture and atherosclerotic inflammation. J Pathol 1997; 181: 93-9.

4. van der Wal AC, Piek JJ, de Boer OJ, et al. Recent activation of the plaque immune response in coronary lesions underlying acute coronary syndromes. Heart 1998; 80: $14-8$.

5. van der Wal AC, Becker AE, van der Loos CM, Das PK. Site of intimal rupture or erosion of thrombosed coronary atherosclerotic plaques is characterised by an inflammatory process irrespective of the dominant plaque morphology. Circulation 1994; 89: 36-44.

6. Kaartinen M, van der Wal AC, van der Loos CM, et al. Mast cell infiltration in acute coronary syndromes: implications for plaque rupture. J Am Coll Cardiol 1998; 32: 606-12.

7. Mach F, Schonbeck U, Sukhova GK, Atkinson E, Libby P. Reduction of atherosclerosis in mice by inhibition of CD40 signaling. Nature 1998; 394 : 200-3.

8. Libby P, Geng YJ, Aikawa M, et al. Macrophages and atherosclerotic plaque stability. Curr Opin Lipidol 1996; 7: 330-5.

9. de Boer OJ, van der Wal AC, Teeling P, Becker AE. Leucocyte recruitment in rupture prone regions of lipid-rich plaques: a prominent role for neovascularization? Cardiovasc Res 1999; 41: 443-9.

10. MachF, Sauty A, Iarossi AS, et al. Differential expression of three Tlymphocyteactivating CXC chemokines by human atheroma-associated cells. J Clin Invest 1999; 104: 1041-50.

11. van der Wal AC, Becker AE, Das PK. Medial thinning and atherosclerosisevidence for involvement of a local inflammatory effect. Atherosclerosis 1993; 103: 55-64

12. Yutani C, Imakita M, Ishibashi-Ueda H, Tsukamoto Y, Nishida N, Ikeda Y. Coronary atherosclerosis and interventions: pathological sequences and restenosis. Pathol Int 1999; 49: 273-90.

13. Bezerra HG, Higuchi ML, Gutierrez PS, Palomino S, Silvestre J, Ramires JAF Atheromas that cause fatal thrombosis are larger and have greater compensatory enlargement than equi-stenotic plaques in the same coronary tree. Cardiovasc Pathol 2001; 10: 189-96.

14. Reis MM, Higuchi ML, Aiello VD, Benvenuti LA. Growth factors in the myocardium of patients with chronic chagasic cardiomyopathy. Rev Soc Bras Med Trop 2000; 33: 509-18.

15. Depre C, Havaux X, Wijns W. Neovascularization in human coronary atherosclerotic lesions. Cathet Cardiovasc Diagn 1996; 39: 215-20.

16. Kumamoto M, Nakashima Y, Sueishi K. Intimal neovascularization in human coronary atherosclerosis: its origin and pathophysiological significance. Human Pathol 1995; 26: 450-6.

17. Zhang Y, Cliff WJ, Schoefl GI, Higgins G. Immunohistochemical study of intimal microvessels in coronary atherosclerosis. Am J Pathol 1993; 143: 164-72.

18. Klagsbrun M, Moses MA. Molecular angiogenesis. Chem Biol 1999; 6: R217-24.

19. Higuchi ML, Castelli JB, Aiello VD, et al. Great amount of C.pneumoniae in ruptured plaque vessel segments at autopsy. A comparative study with stable plaques. Arq Bras Cardiol 2001; 74: 149-51.

20. Smits PC, Pasterkamp G, de Jaegere PP, de Feyter PJ, Borst C. Angioscopic complex lesions are predominantly compensatory enlarged: an angioscopy and intracoronary ultrasound study. Cardiovasc Res 1999; 41: 458-64.

21. Mao D, VanVickle SJ, Curci JA, Thompson RW. Expression of matrix metalloproteinases and TIMPs in human abdominal aortic aneurysms. Ann Vasc Surg 1999; 13: 236-7.

22. Thompson RW, Parks WC. Role of matrix metalloproteinases in abdominal aortic aneurysms. Ann N Y Acad Sci 1996; 800: 157-74.

23. Galis ZS, Sukhova GK, Lark MW, Libby P. Increased expression of matrix metalloproteinases and matrix degrading activity in vulnerable regions of human atherosclerotic plaques. J Clin Invest 1994; 94: 2493-503.

24. Beckman EN. Plasma cell infiltrates in atherosclerotic abdominal aortic aneurysms. Am J Clin Pathol 1986; 85: 21-4.

25. Juvonen J, Juvonen T, Laurila A, et al. Demonstration of Chlamydia pneumoniae in the walls of abdominal aortic aneurysms. J Vasc Surg 1997; 25: 499-505.

26. Schonbeck U, Mach F, Sukhova GK, et al. CD40 ligation induces tissue factor expression in human vascular smooth muscle cells. Am J Pathol 2000; 156: 7-14.

27. Bezerra HG, Higuchi ML, Gutierrez PS, Ramires JAF. Do bypassed coronary atheromas present histopathological features of instability? J Am Coll Cardiol 2002, Abstract. 